\title{
Visual Representations in Knowledge Management: \\ Framework and Cases
}

\section{Research paper}

\begin{abstract}
The purpose of this article is to explore the potential of visualization for corporate knowledge management. The employed methodology consists of a taxonomy of visualization formats that are embedded in a conceptual framework to guide the application of visualization in knowledge management according to the type of knowledge that is visualized, the knowledge management objective, the target group, and the application situation. This conceptual framework is illustrated through real-life examples. Our findings show that there is much room for knowledge management applications based on visualization beyond the mere referencing of experts or documents through knowledge maps. The research implications thus consist of experimenting actively with new forms of visual knowledge representation and evaluating their benefits or potential drawbacks rigorously. In terms of practical implications, the authors encourage managers to look beyond simple diagrammatic representations of knowledge and explore alternative visual languages, such as visual metaphors or graphic narratives. The originality and value of this paper consists of two elements: first, the systematic, descriptive and prescriptive approach towards visualization in knowledge management, and second the innovative examples of how to harness the power of visualization in knowledge management
\end{abstract}

Key Words: knowledge visualization, knowledge creation, knowledge transfer, diagrams, maps, visual metaphors

\section{Introduction}

Making knowledge visible so that it can be better accessed, discussed, valued or generally managed is a long standing objective in knowledge management (Sparrow 1998). Knowledge maps, knowledge cartographies, or knowledge landscapes are often heard terms that are nevertheless rarely defined, described or demonstrated. In this contribution, the authors review the state-of-the-art in the area of knowledge visualization and describe its background and perspec- 
tives. We define the concept and differentiate it from other approaches, such as information visualization or visual communication. Core knowledge visualization types, such as conceptual diagrams or visual metaphors, are distinguished and examples of their application in business are shown and discussed. Implications for research and practice are summarized and future trends in this domain are outlined.

The emerging field of knowledge visualization examines the use of visual representations to improve the management of knowledge on all levels (personal, interpersonal, team, organizational, inter-organizational, and societal). Knowledge visualization designates all graphic means that can be used to construct, assess, measure, convey or apply knowledge (i.e., complex insights, experiences, methods, etc.). Beyond the mere transport of information or facts, people who employ knowledge visualization aim to create, assess, reference or transfer insights, experiences, attitudes, values, expectations, perspectives, opinions and predictions, and this in a way that enables someone else to reconstruct, remember, find or apply these insights correctly. Examples of knowledge visualization in this understanding are insightful graphic formats such as heuristic sketches (e.g. the ad-hoc, joint drawings of complex ideas in meetings), conceptual diagrams (such as Porter's Five Forces diagram), visual metaphors (such as an iceberg visualization distinguishing implicit and explicit forms of knowledge), or knowledge maps (such as a landscape of in-house experts).These graphic formats capture not just (descriptive) facts or numbers, but 
contain also prescriptive and prognostic insights, principles, basic assumptions and relations. They are used as communication devices in order to trigger sense making activities and to motivate viewers to re-construct meaning. Thus, the 'what' (object), the 'why' (purpose), and the 'how' (methods) of knowledge visualization differ from information visualization. These application questions are systematically addressed in a framework presented in the next section.

\section{A Framework for Knowledge Visualization}

For the effective creation and transfer of knowledge through visualization, at least five perspectives should be considered. These perspectives answer five key questions with regard to visualizing knowledge, namely:

1. What type of knowledge is visualized (content)?

2. Why should that knowledge be visualized (purpose, km process)?

3. For whom is the knowledge visualized (target group)?

4. In which context should it be visualized (communicative situation: participants, place/media)?

5. How can the knowledge be represented (method, format)?

Listing possible answers to these key questions leads to a conceptual framework for visual representations in knowledge management that can provide an overview of the knowledge visualization field and guide its application (see Figure 1). The framework depicted in figure 1 thus answers the following question: Why do we show what to whom in which knowledge-related situation and how? The individual elements of this question are discussed briefly below. 
The knowledge type perspective can be used to identify the type of knowledge that has to be transferred. The framework distinguishes among six types of knowledge: declarative knowledge (know-what), procedural knowledge (knowhow), experiential knowledge or experience (know-why), people-related knowledge (know-who), orientation or location-based knowledge (know-where), scenario-based knowledge (know-what-if) or normative, value-based knowledge. For a similar distinction see for example Alavi \& Leidner 2001.

With the help of the visualization goal perspective one can distinguish among several reasons why visual knowledge representations are used in knowledge management. Motives for knowledge visualization use are knowledge sharing through visual means, knowledge creation, learning from visual representations, codifying past experiences visually for future users, or mapping knowledge (Vail 1999) so that experts, for example within a large organization, can be more easily identified.

The target group perspective emphasizes the fact that the visualization of knowledge must accommodate the preferences of the primary and potential target groups. Their prior knowledge, their expectations and time constraints must be taken into account when choosing a visualization format. Managers for example may be more comfortable analyzing a quantitative diagram, whereas the communication to employees might involve more playful formats such as visual metaphors. With regard to visualizations that need regular updating, improve- 
ments or maintenance (such as online knowledge maps) the group responsible for such updating or medications tasks must also be taken into account (particularly with regard to their technological abilities). Otherwise a knowledge visualization may quickly become outdated due to the inadequate maintenance by its target groups.

The visualization situation perspective stresses the fact that the use of visualization depends on the physical or virtual setting and on the number of people that interact to manage knowledge. A large virtual community who shares experiences on a public website needs a different kind of knowledge visualization, than a top management team who meets in order to assess the distinctive capabilities of their corporation.

The visualization format perspective structures the visualization formats into seven main groups: structured text/tables, mental (non-material) visualization and visual story telling, heuristic sketches, conceptual diagrams/concept maps, visual metaphors, knowledge maps, and graphic interactive environments. These visualization types are based on prior visualization taxonomies and use the media prerequisites as a classification criterion (from comparatively little media requirements for text and sketches to extensive multimedia requirements for interactive visual environments). 


\section{Take in Figure 1}

Having given an overview of the main formats of knowledge visualization, each of the seven visual styles will be more closely examined. The next section will outlined how these formats can be matched with adequate knowledge types and applied for specific application contexts.

\section{Examples of Knowledge Visualization}

\section{Structured text and tables: Organizing knowledge with grids}

Visually structured texts or numbers are a first visualization type. A first step is the formatting of text, for example through highlighting words, formatting paragraphs, using different colors, fonts and font sizes. A second step is the integration of textual items into superimposed visual structures, such as a tree structure or a table. Tables are grid-like arrangements of textual information that can be used for matching, listing, comparison, or rating purposes. Typical table applications in knowledge management range from database overviews to expert directories that follow the table format. Another type of visually structured text are visually enhanced results or search result visualizations. Here search algorithms are combined with visual clues, such as highlighted keywords in texts or relevance ranking bars. Examples for such systems are Envision ( Fox et al. 1993; Fox et al. 2002) or Gridvis (Weiss-Lijn et al. 2001). An overview of such systems is presented by (Nowell et al. 1996; Börner \& Chen 2002). Structured text can also relate to the layout of printed or on-screen pages. One possible metho- 
dology to label and structure text is Robert Horn's information mapping method (Horn 1998).

\section{Heuristic sketches: The ad-hoc depiction and creation of insight}

Heuristic Sketches are drawings that are used to assist the personal or group reflection and communication process by making knowledge-in-progress explicit and debatable. Generally a sketch can be defined as "a rough drawing or painting in which an artist notes down his preliminary ideas for a work that will eventually be realized with greater precision and detail." In the context of knowledge management, these drawings can be called heuristic sketches to highlight their problem solving potential. The main benefits of heuristic sketches are as follows: (1) They represent the main idea and key features of a preliminary insight. (2) They are flexible and highly accessible because they are accompanied by explanations and developed jointly. (3) They are fast and help to quickly visualize emergent notions. (4) The use of a pen on a flipchart attracts the attention towards the communicator. (5) Heuristic sketches allow room for one's own interpretations and foster the creativity in groups. Sketches can also be used to capture mental models of individuals with the goal to get insights on how people perceive reality and think about a concept. Examples of this are urban planners who sometimes try to capture the perceptions of a city by asking individuals to sketch their mental map of a city. Kevin Lynch (Lynch 1960) found that in doing so people use only five different types for the mental mapping: "Districts" are regions (e.g. neighborhoods); "edges" are boundaries between 
regions (e.g. river); "paths" are the channels along which people move (e.g., street); "nodes" are points to and from which people travel (e.g., station); and "landmarks" are orientational points (e.g. skyscraper). A real-life example of sketches that facilitate knowledge transfer is the hand-drawing library for client advisors used by the Private Banking division of a multinational bank. In order to be able to quickly explain important relationships or financial products, the client advisors have memorized a set of sketches from an especially prepared sketchbook. In explaining financial issues to clients, the advisors can develop these sketches on the table and thus highlight and discuss slightly complex points in a more salient and persistent way. The client in return can pinpoint issues in the sketch that he has not understood or wants to have elaborated further. Through the flexible and rapid format of sketches, abstract concepts are thus turned into accessible elements that can be more easily shared and discussed. New IT tools such tablet PCs, mind mapping, concept mapping or sketching software support digital sketching for rapid knowledge creation and depiction.

Conceptual Diagrams: The Elicitation of Implicit Knowledge through Templates

Conceptual Diagrams as seen in Figure 2 are schematic depictions of abstract ideas with the help of standardized shapes (such as arrows, circles, pyramids or matrices). They are used to structure information and illustrate relationships. For 
the transfer and creation of knowledge conceptual diagrams help to make abstract concepts accessible, to reduce the complexity to the key issues, to amplify cognition and to discuss relationships.

\section{Take in Figure 2}

An example of a particularly knowledge-intensive conceptual diagram is the Toulmin chart, based on the argumentation theory of Steven Toulmin (Toulmin 1958). Such a chart helps to break down an argument into different parts (such as claim, reasons, and evidence) which is useful when evaluating the validity of a claim. In the case example below, we have used the Toulmin diagram to make implicit knowledge regarding a product explicit in a diagnostics development team and thus improve knowledge integration in the team (Figure 3).

\section{Take in Figure 3}

Concept Maps Âhlberg 2004; Cañas et al. 2005; Tergan 2005) are another form of diagrams and also use diagrammatic representations to visually reference knowledge or for visualizing the relations among concepts. A concept map generally consists of two elements: An item and a relationship between two items. Concept maps illustrate both an overview and detail, and interrelationships among these details. Concept Map are helpful for different learning and communication tasks: (1) To brainstorm or summarizing contents, (2) for sense making by illustrating and overview and details, (3) for structuring digital in- 
formation, (4) as visual interface to databases, (5) for shared understanding of contents.

An example of the use of diagrams in knowledge management is the following: A large European market research company has developed an interactive diagram to be used on its intranet knowledge portal (in one country organization of the group). The diagram (Figure 4) gives an overview of most of the company's tools, techniques and methods along the central value chain (the process cycle in the middle). By clicking on an item in the diagram, employees can access further descriptions, guidelines, templates, examples or pointers to experts on the tool or method.

\section{Take in Figure 4}

\section{Visual Metaphors: Relating Domains to Improve Knowledge Transfer}

A metaphor provides the path from the understanding of something familiar to something new by carrying elements of understanding from the mastered subject to a new domain. This is why Aristotle calls the metaphor a tool of cognition. A metaphor provides rapid information, is highly instructive, and facilitates the process of learning. As Worren et al. (2002) have pointed out, metaphors can also improve memorability and coordination in groups. Visual metaphors used for knowledge transfer or creation can either be natural objects or phenomena (e.g., mountains, icebergs, tornado) or artificial, man-made objects (e.g., a bridge, a ladder, a temple), activities (e.g., climbing, etc.), or concepts (e.g., war, 
family). Their main feature is that they organize information meaningfully. In doing so, they fulfill a dual function: first, they position information graphically to organize and structure it. Second, they convey an implicit insight about the represented information through the key characteristics (or associations) of the metaphor that is employed. In Figure 5 the metaphor of a bridge was used in a corporate training context to convey how to lead successful negotiations.

\section{Take in Figure 5}

\section{Knowledge Maps: Charting Knowledge Application and Navigating Expertise}

Knowledge maps (Eppler 2002; Burkhard et al. 2005) are graphic formats that follow cartographic conventions to reference relevant knowledge. A knowledge map generally consists of two parts: a ground layer which represents the context for the mapping (such as a city), and the individual elements that are mapped within this context (e.g., streets). The ground layer typically consists of the mutual context that all employees can understand and relate to, such as a business model, a product, the competency areas, or a geographic map. The elements which are mapped onto such a shared context range from experts and communities of practice to more explicit and codified forms of knowledge such as articles, patents, lessons learned bases, or expert systems. Knowledge maps are thus graphic directories of knowledge-sources, -assets, -structures, -applications, or -development stages. 
In Figure 6 the customized Tube Map visualization (Burkhard \& Meier 2005) illustrates a five year quality development project. The subway-lines represent individual target groups and the stations milestones. The knowledge map was printed on a poster (2,4 x 1,2 meters) and located in front of an elevator to foster creativity and initiate discussion. Two evaluations can be found in Burkhard and Meier (2005) and Burkhard et al. (Burkhard et al. 2005). An automatic layout algorithm for Project Tube Map is discussed in Stott et al. (Stott et al. 2005).

\section{Take in Figure 6}

Another type for mapping knowledge are knowledge domain structures. Knowledge domain structures focus on identifying and visually representing the dynamics of scientific frontiers in a multidisciplinary context and allow new ways of accessing knowledge sources (such as authors, institutions, papers, journals, etc.) by visualizing linkages, relationships, and structures of knowledge domains (Chen 1998; 2000; 2003).

\section{Interactive Visualizations and Animations: Exploring Complexity}

Interactive Visualizations are computer-supported interactive visualizations that allow users to control, interact, and manipulate different types of information in a way that fosters the transfer and creation of knowledge. By interacting with the information, new insights are created or shared. Interactive Visualizations help to fascinate and focus people, to enable interactive collaboration and persistent 
conversations, and to illustrate, explore, and discuss complex issues in various contexts. In the Infoticle application (Vande Moere et al. 2004) the animation of data-driven particles (Infoticles) helps to explore large time-varying datasets and allows seeing the behaviour of individual data entries in the global context of the whole dataset.

In similar ways, the interactive parameter ruler (Figure 7) enables teams and individuals to explore alternatives in real-time through sliders in the ruler application. As they enter evaluation criteria or decision options and move them into various positions, participants develop a common understanding regarding a complex issue. The joint visual interaction is thus a catalyst for collective knowledge development and transfer in groups.

\section{Take in Figure 7}

Animations show a predefined sequence of visual images as in a movie. An example for an animation that is used for intercultural knowledge transfer are the safety instruction videos in airplanes. Animations can also be interactive; then the user has the possibility to choose at certain points of the animation how the animation continues. Another type of interactive visualizations that can be used for knowledge management purposes are geographic information systems (GIS) (such as Google Earth), virtual environments (such as Habo Hotel ${ }^{1}$ ) or computer games (MUDs). The last two can be used for knowledge sharing in virtual communities of practice. 


\section{Resulting Application Areas of Visualization in Knowledge Management}

Knowledge visualization formats as the ones reviewed in the previous section can help to solve several predominant, knowledge-related problems in organizations. This section provides a summary of how visualization can be used to facilitate knowledge transfer, creation, identification, evaluation, and application.

A first application area regards the omnipresent problem of knowledge transfer (i.e., knowledge asymmetry). Knowledge visualization offers a systematic approach to transfer knowledge at various levels: among individuals, from individuals to groups, between groups, and from individuals and groups to the entire organization. To do so, knowledge must be recreated in the mind of the receiver (El Sawy et al. 1997). This depends on the recipient's cognitive capacity to process the incoming stimuli (Vance \& Eynon 1998). Thus, the person responsible for the transfer of knowledge not only needs to convey the relevant knowledge at the right time to the right person, he or she also needs to convey it in the right context and in a way that it can ultimately be used and remembered. Graphics such as rich, but easily understandable visual metaphors can serve this purpose, as the brain can process images more easily than text. In this context, visualization can also facilitate the problem of inter-functional knowledge transfer, i.e., the communication among different stakeholders and experts with different professional backgrounds. Visual methods for the transfer complex knowledge are thus one emergent sub-discipline within knowledge visualization.

\footnotetext{
${ }^{1}$ http://www.habbohotel.com/
} 
Another application area of visualization within knowledge management is knowledge creation.

Knowledge visualization offers great potential for the creation of new knowledge in groups, thus enabling innovation. Knowledge visualization offers methods to use the creative power of imagery and the possibility of fluid rearrangements and changes. It inspires and enables groups to create new knowledge, for instance by use of heuristic sketches. Unlike text, these ad-hoc graphic formats can be quickly and collectively changed and thus propagate the rapid and joint improvement of ideas. They also capture more implicit aspects of personal knowledge (Polanyi 1958) that cannot be expressed easily through verbal means, but rather shown through graphic analogies or symbols.

Yet another application area for visualization in knowledge management is knowledge identification. Knowledge maps (Eppler 2002) have been used for a while to map the expertise located within a particular company and link it to personal homepages of specific experts. Such maps can provide an overview on various forms of knowledge sources, such as experts, documents, project teams, organizations or even patents.

Knowledge visualization can also help to evaluate, rate and measure knowledge. Next to identifying relevant knowledge, visualization can be employed to facilitate the process of evaluating knowledge assets. By providing conceptual diagrams as interactive graphic frameworks and multi-dimensional scales as communication support, knowledge can be jointly assessed and evaluated and weak spots or core competencies can be detected. 
A further area where visualization can add value to knowledge management is knowledge application. In this context it is vital that individuals can use the documented explicit knowledge of others and are not overloaded by it. Visualization can be used as an effective strategy against information overload: Information overload is a major problem in knowledge-intensive organizations. Knowledge visualizations help to compress large amounts of reasoned information with the help of interactive visualization, i.e., graphic models and simulations that absorb complexity and render it accessible through easy-to-use manipulation. This can be a vital prerequisite for the three application domains mentioned previously (transfer, creation, and communication).

A final, often neglected area of knowledge management, where visualization can play a pivotal role is knowledge marketing. Through the help of appealing visuals abstract competence can be converted into tangible value propositions. In order to market their skills and experiences, companies rely not only on symbols, such as knowledge brands, but also use visual representations of their knowledge to signal competence. Knowledge maps and visual metaphors seem particularly apt for this purpose as they make new material accessible through familiar structures.

Summarizing these application examples one can qualify the fit of the various visualization formats for different knowledge management application areas, as shown in the diagram below. 


\section{Take in Figure 8}

The table reveals that knowledge visualization is a particularly suited strategy for knowledge creation (through its creative potential) and for knowledge transfer (as it is often a joint construction activity). Dark areas designate visualization formats that, in our experience, are well suited to support the respective knowledge management task. Light grey areas designate other feasible combination between visualization formats and knowledge management processes. Future experiments, case studies and tool evaluations can be used to test the stipulated relationships that are now based on preliminary findings from select field tests.

\section{Conclusion}

In this paper, we have highlighted the functional role of graphic representations for various knowledge management processes. We have distinguished among different formats of knowledge visualization and we have related these to different application parameters in a conceptual framework. Examples of such application contexts have been presented in order to highlight which formats serve which kind of function.

In terms of future trends, knowledge visualization will evolve with regard to new formats and new application areas. The potential to combine various formats (such as diagrams, maps, and metaphors) in a complementary way (as architects use them) seems promising. It also seems clear that knowledge visualiza- 
tion will be used in other settings than just the traditional computer desktop environment. Examples of new application areas for knowledge visualization can be found, for example, in the visual communication of corporate missions, strategies, value propositions, and business scenarios. New applications can also be envisioned by combining knowledge visualization with other innovative approaches in knowledge management, such as story telling. Storytelling is a closely related knowledge management tool, as it strives for rich, mental imagery (Loebbert 2003). The authors believe that stories can be combined with knowledge visualization formats (as in visualized story trails) to trigger and accelerate the creation and dissemination of knowledge in organizations. 


\section{References}

Âhlberg, M. (2004), "Varieties of concept mapping. Concept maps: Theory, methodology, technology." Proceedings of the first international conference on concept mapping, Universidad Pública de Navarra, Pamplona, Spain.

Alavi, M., Leidner, D. (2001), "Knowledge Management and Knowledge Management Systems: Conceptual Foundations and Research Issues." MIS Quarterly, Vol 25 No 1, pp. 107-136.

Börner, K., Chen, C. (2002) "Visual Interfaces to Digital Libraries: Motivation, Utilization, and Socio-Technical Challenges". Lecture Notes in Computer Science. 2539. Springer Verlag, New York, pp. 1-12.

Burkhard, R., Meier, M. (2005), "Tube Map Visualization: Evaluation of a Novel Knowledge Visualization Application for the Transfer of Knowledge in Long-Term Projects." Journal of Universal Computer Science, Vol. 11 (4) pp. 473-494.

Burkhard, R., Meier, M., Rodgers, P., Smis, M., Stott, J. (2005), "Knowledge Visualization: A Comparative Study between Project Tube Maps and Gantt Charts." I-Know '05, Graz, Austria, pp. 388-395.

Burkhard, R., Meier, M., Smis, M., Allemang, J., Honisch, L. (2005), "Beyond Excel and Powerpoint: Knowledge Maps for the Transfer and Creation of Knowledge in Organizations." Ninth International Conference on Information Visualization (IV05), London. 
Cañas, A. J., Carff, R., Hill, G., Carvalho, M., Arguedas, M., Eskridge, T. C., Lott, J., Carvajal, R. (2005), "Concept Maps: Integrating Knowledge and Information Visualization." In: Tergan, S.-O., Keller, T. (eds.): Knowledge and Information Visualization, LNCS 3426. Springer-Verlag, Berlin Heidelberg, pp. 205-219.

Chen, C.: "Bridging the Gap: The Use of Pathfinder Networks in Visual Navigation." Journal of Visual Languages and Computing, Vol. 9 (3) (1998) pp. 267-286.

Chen, C.: "Domain Visualization for Digital Libraries." International Conference on Information Visualisation (IV2000), London, England, 19-21 July 2000 (2000) pp. 261-267.

Chen, C. (2003), Mapping Scientific Frontiers: The Quest for Knowledge Visualization. Springer, London.

El Sawy, O. A., Eriksson, I., Carlsson, S., Raven, A. (1997), "Understanding the Nature of Shared Knowledge Creation Spaces Around Business Processes: An International Investigation." Carnegie Bosch Institute Conference on Knowledge in International Corporations, Rome, Italy.

Eppler, M. J. (2002), "Making Knowledge Visible through Knowledge Maps: Concepts, Elements, Cases", in Holsapple, C. W.(Ed.) Handbook on Knowledge Management, Volume 1: Knowledge Matters. Springer, Berlin, pp. 189-206.

Fox, E. A., Hix, D., Nowell, L. T., Brueni, D. J., Wake, W. C., Heath, L. S., Rao, D. (1993), "Users, user Interfaces, and objects: Envision, a digital li- 
brary." Journal of the American Society for Information Science, Vol. 44 (8), pp. $480-491$.

Fox, E. A., North, C., Wang, J., Abhishek, A., Anil, B., Supriya, A. (2002),

"Enhancing the ENVISION interface for digital libraries", Second ACM/IEEE-

CS joint conference on Digital libraries, ACM Press, Portland (OR), 14-18 June 2002, pp. $275-276$

Horn, R. E. (1998), Visual Language: Global Communication for the 21st Century. MacroVU Press, Bainbridge Island (WA).

Loebbert, M. (2003), Storymanagement. Klett-Cotta, Stuttgart.

Lynch, K. (1960), The image of the city. MIT Press, Cambridge.

Nowell, L. T., France, R. K., Hix, D., Heath, L. S., Fox, E. A. (1996), "Visualizing search results: some alternatives to query-document similarity", 19th Annual international ACM SIGIR Conference on Research and Development in Information Retrieval (SIGIR96), Zurich, Switzerland, 18-22 August 1996, pp. 67-75.

Polanyi, M. (1958), Personal Knowledge: towards a post-critical philosophy. Routledge, London.

Sparrow, J. (1998), Knowledge in Organizations: Access to Thinking at Work. Sage Publications Ltd, Thousand Oaks (CA).

Stott, J. M., Rodgers, P., Burkhard, R., Meier, M., Smis, M. T. J. (2005), "Automatic Layout of Project Plans Using a Metro Map Metaphor", Ninth International Conference on Information Visualization (IV05), London. 
Tergan, S.-O. (2005), "Digital Concept Maps for Managing Knowledge and Information", in: Tergan, S.-O., Keller, T. (eds.): Knowledge and Information Visualization. LNCS 3426. Springer-Verlag, Berlin Heidelberg, pp. 185-204.

Toulmin, S. E.(1958), The Uses of Argument. Cambridge University Press, Cambridge.

Vail, E. F. (1999), "Mapping Organizational Knowledge: Bridging the business-IT communication gap", Knowledge Management Review, No. 8, pp. $10-15$.

Vance, D. A., Eynon, J. (1998), "On the Requirements of Knowledge Transfer Using Information Systems: A Schema Whereby Such Transfer Is Enhanced", 4th AIS Americas Conference on Information Systems, Baltimore (MD), 632-634.

Vande Moere, A., Mieusset, K. H., Gross, M. (2004), "Visualizing Abstract Information using Motion Properties of Data-Driven Particles", Conference on Visualization and Data Analysis 2004, IS\&T/SPIE Symposium on Electronic Imaging 2004, San Jose (CA),

Weiss-Lijn, M., McDonnell, J. T., James, L. (2001), "Visualising Document Content with Metadata to Facilitate Goal-directed Search", 5th International Conference on Information Visualization (IV01), London, 25-27 July 2001, pp. 71-76.

Worren, N. A., Moore, K., Elliott, R. (2002), "When theories become tools: Toward a framework for pragmatic validity", Human Relations, Vol 55 No 10, pp. $1227-1250$. 


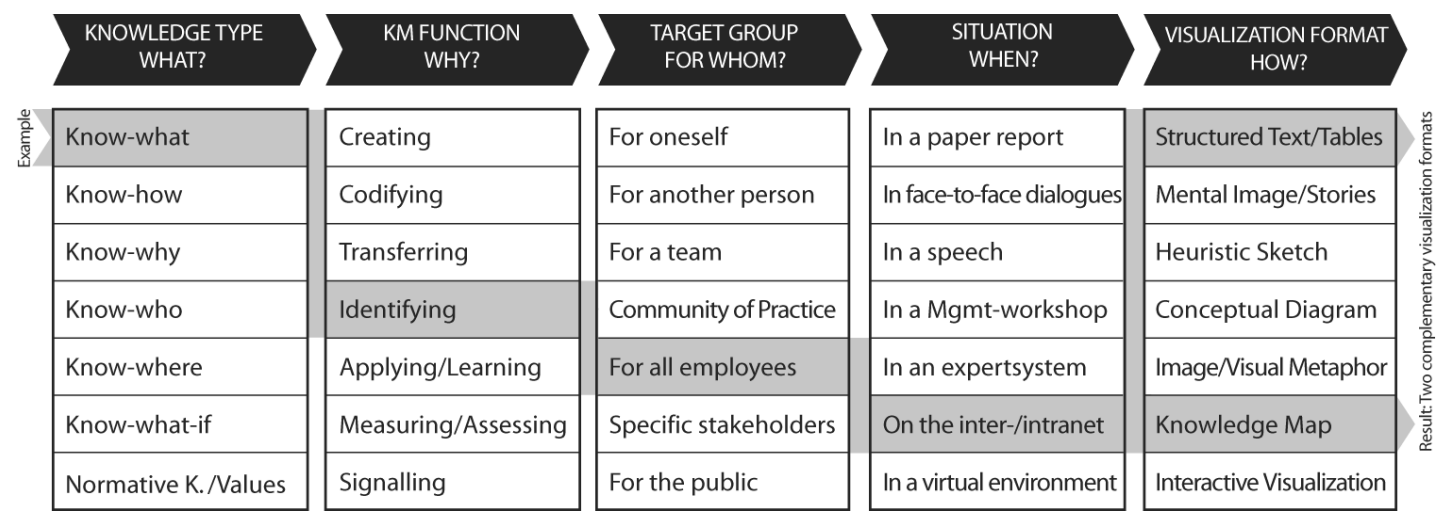

Fig. 1. A Framework for the Use of Visualization in Knowledge Management

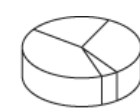

PIECHART

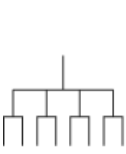

TREE

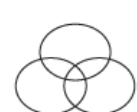

VENN<smiles>C#CC[SH](CC)CC</smiles>

MINDMAP

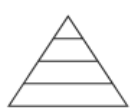

PYRAMID

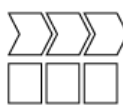

PROCESS

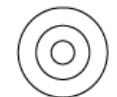

CIRCLES

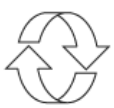

CYCLE

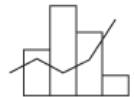

BARS, LINES

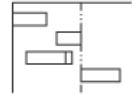

GANTT
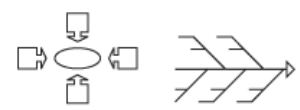

FIVE FORCES ISHIKAWA
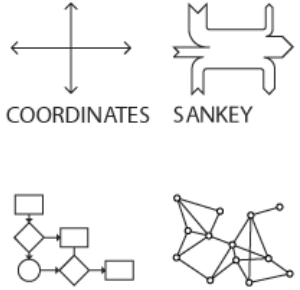

COORDINATES SANKEY

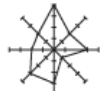

RADAR/KIVIAT

FLOWCHART NETWORK SYNERGY

Fig. 2. An overview of frequently used conceptual diagrams

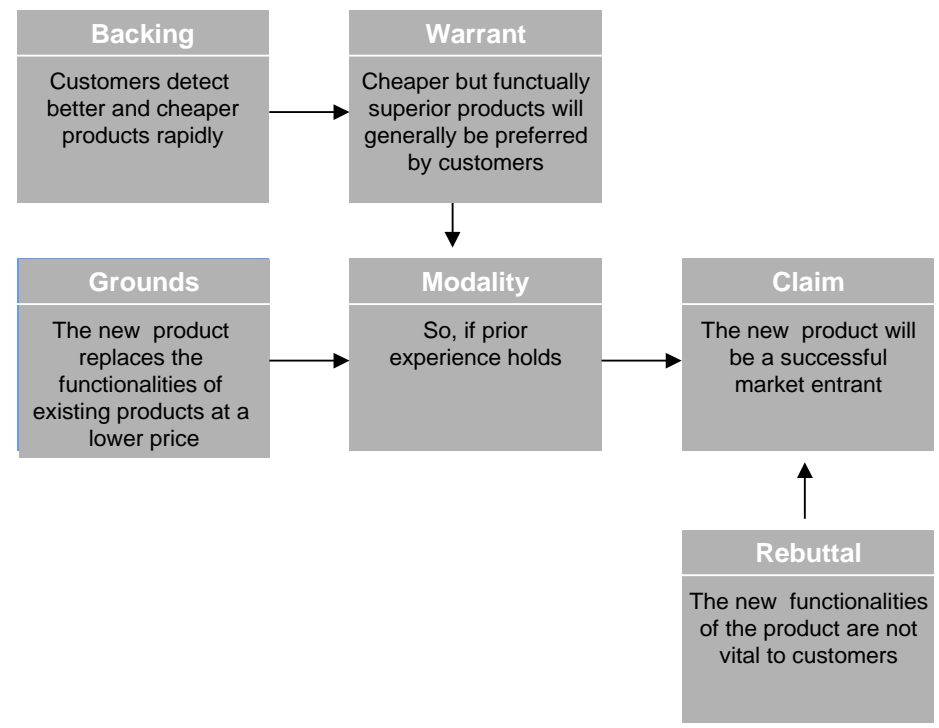

Fig. 3. A Toulmin diagram used to elicit basic assumptions and beliefs of team members 


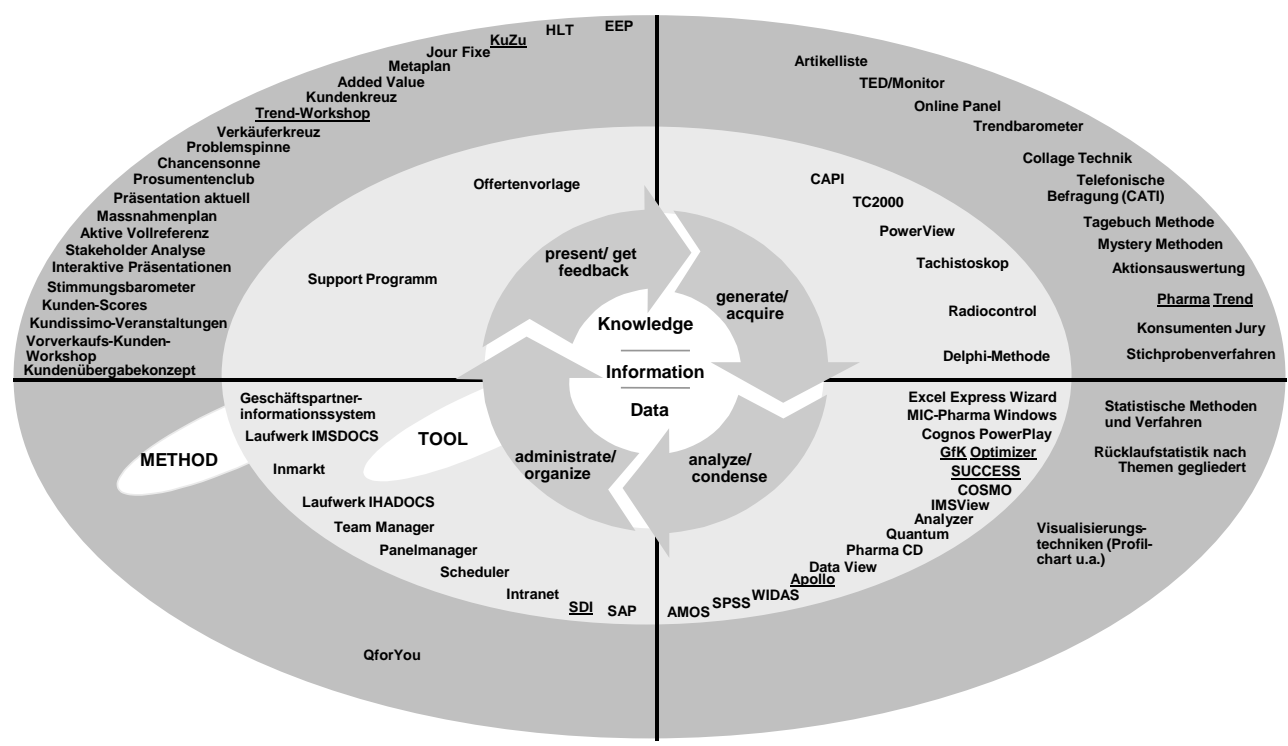

Fig. 4. A knowledge diagram referencing methodological know-how of a mar-

ket research company

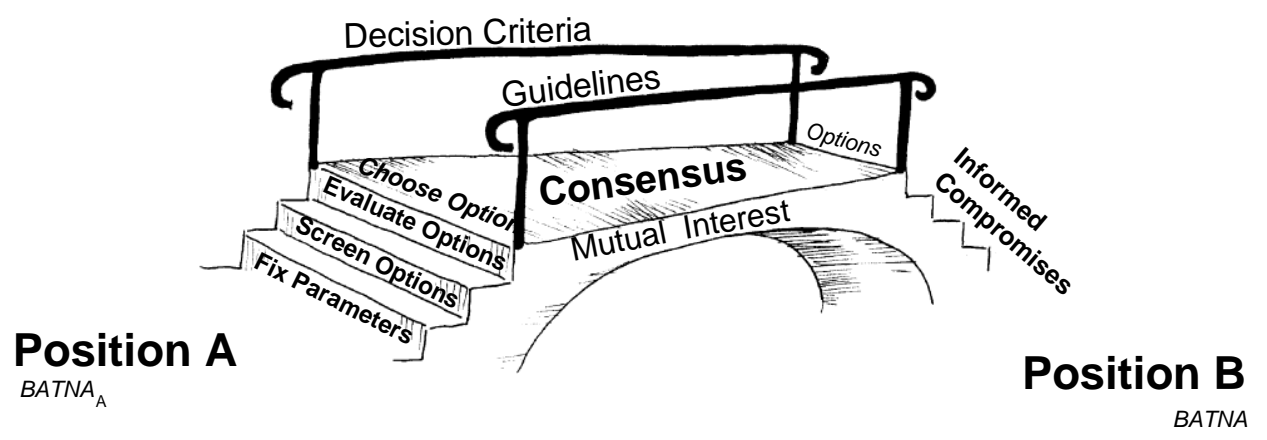

Fig. 5. A visual metaphor that outlines procedural knowledge on negotiation 

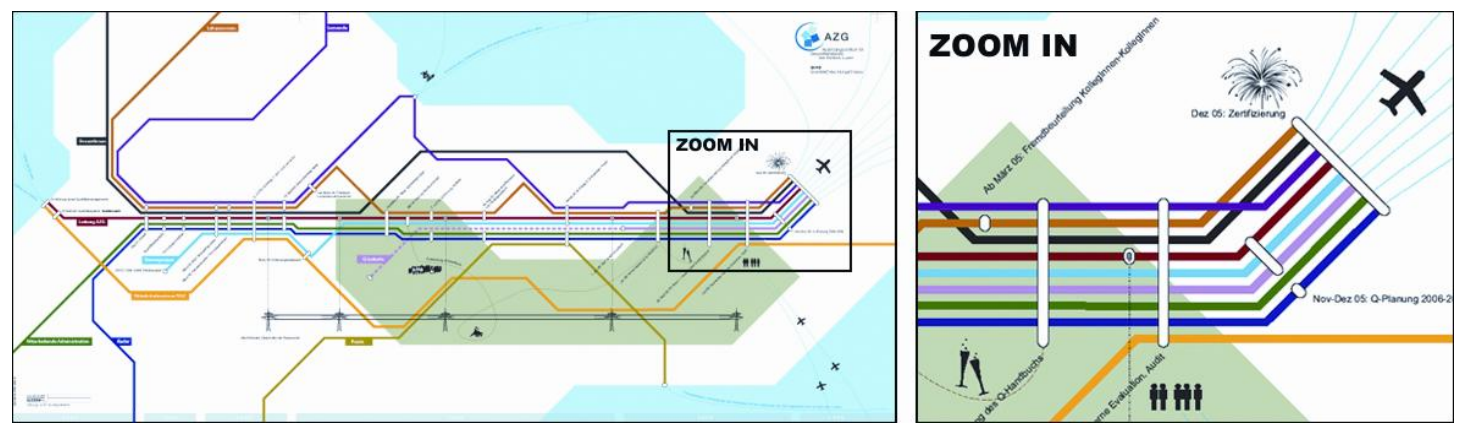

Fig 6. A tube map diagram visualizes procedural project knowledge

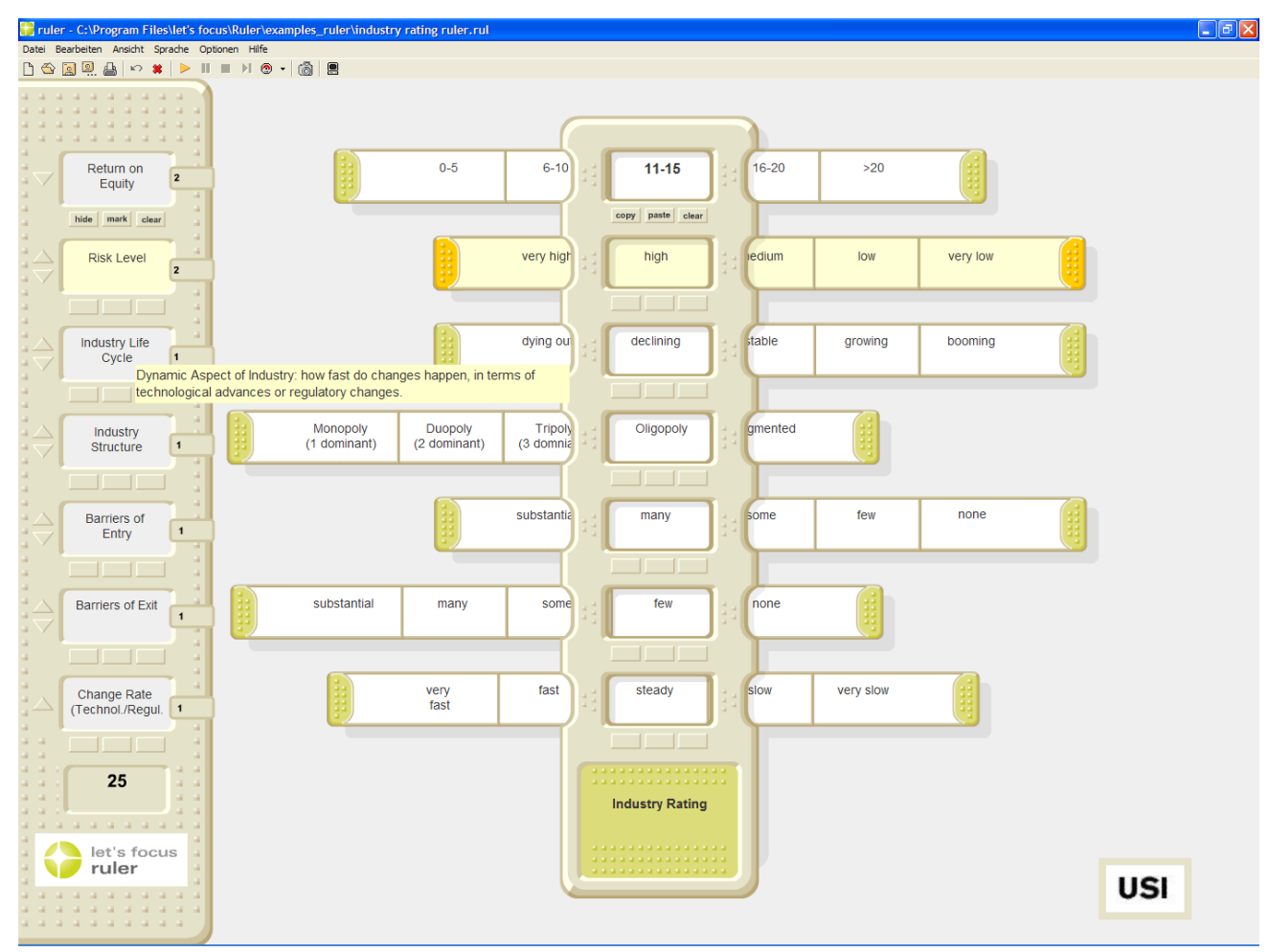

Fig. 7. An interactive ruler that enables teams to integrate judgments, assessments, and evaluations in groups in real-time 


\begin{tabular}{|l|c|c|c|c|c|c|}
\hline Formats KM Process & Creation & Codification & Transfer & Identification & Application & Measurement \\
\hline Structured Text/Tables & & $\checkmark \checkmark$ & $\checkmark$ & & & $\checkmark$ \\
\hline Mental Image/Stories & $\checkmark$ & & $\checkmark \checkmark$ & & $\checkmark \checkmark$ & $\checkmark \checkmark$ \\
\hline Heuristic Sketch & $\checkmark \checkmark$ & & $\checkmark$ & & $\checkmark$ & \\
\hline Conceptual Diagram & $\checkmark$ & $\checkmark \checkmark$ & $\checkmark$ & & & \\
\hline Image/Visual Metaphor & $\checkmark$ & & $\checkmark \checkmark$ & & & \\
\hline Knowledge Map & $\checkmark$ & & & $\checkmark \checkmark$ & & \\
\hline Interactive Visualization & $\checkmark \checkmark$ & & & & $\checkmark \checkmark$ & \\
\hline
\end{tabular}

Figure 8: Visualization formats for different knowledge management tasks 